\title{
The continued emergence of hantaviruses: isolation of a Seoul virus implicated in human disease, United Kingdom, October 2012
}

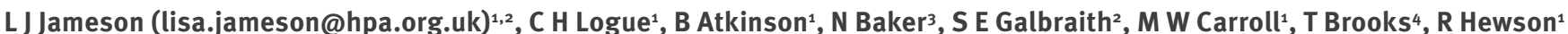

1. Virology and Pathogenesis, Microbiology Services, Health Protection Agency, Porton Down, Wiltshire, United Kingdom

2. Department of Clinical Infection, Microbiology and Immunology, Institute of Infection and Global Health, University of Liverpool, Liverpool, United Kingdom

3. National Collection of Pathogenic Viruses, Microbiology Services, Health Protection Agency, Porton Down, Wiltshire, United Kingdom

4. Rare and Imported Pathogens Laboratory, Microbiology Services, Health Protection Agency, Porton Down, Wiltshire, United Kingdom

Citation style for this article:

Jameson LJ, Logue CH, Atkinson B, Baker N, Galbraith SE, Carroll MW, Brooks T, Hewson R. The continued emergence of hantaviruses: isolation of a Seoul virus implicated in human disease, United Kingdom, October 2012. Euro Surveill. 2013;18(1):pii=20344. Available online: http://www.eurosurveillance.org/ViewArticle. aspx?Articleld=20344

Article submitted on 04 December 2012 / published on 03 January 2013

Following a suspected case of hantavirus in a patient suffering from acute kidney injury, rodents from the patient's property in Yorkshire and the Humber, United Kingdom (UK) were screened for hantaviruses. Hantavirus RNA was detected via RT-PCR in two Rattus norvegicus. Complete sequencing and phylogenetic analysis established the virus as a Seoul hantavirus, which we have provisionally designated as strain Humber. This is the first hantavirus isolated from wild rodents in the UK and confirms the presence of a pathogenic Seoul virus in Europe.

In January 2012, as part of routine diagnostic services, the Rare and Imported Pathogens Laboratory (RIPL) at the Health Protection Agency (HPA) Porton, detected a suspected case of hantavirus infection in a patient diagnosed with acute kidney injury (AKI) from Yorkshire and the Humber, United Kingdom (UK). Positive serology by way of indirect immunofluorescence (Euroimmun, Germany) showed evidence of hantavirus antibodies specific to Hantaan virus (HTNV) and Seoul virus (SEOV) with rising IgG titres $>1: 10,000$. The patient disclosed regular exposure to rodents at their home and noted that the rat population had increased in recent months. With permission from the patient, trapping of rodents in the vicinity of the family residence and farm was undertaken in February/ March 2012, with the aim of confirming the presence of an aetiological agent in these rodents that might be responsible for the patient's AKI.

\section{Rodent sampling}

Permission was obtained from the patient and family to trap rodents at their residence. Between 28 February and 1 March 2012, rodents were trapped using a Longworth trap (Penlon Ltd., Oxford) or snap traps and, where necessary, humanely killed via cervical dislocation before being flash frozen on dry ice.
Eleven rodents were analysed for the presence of hantavirus RNA: five Apodemus sylvaticus (wood mouse), four Rattus norvegicus (Norway rat) and two Myodes glareolus (bank vole). Following tissue harvest by necropsy, lung tissue was homogenised and total RNA was extracted using QIAamp viral RNA mini kit (Qiagen). RNA from each rodent lung was subjected to a genusspecific RT-PCR assay using hantavirus primers targeting the $S$ segment [1]. Two $R$. norvegicus ( $\mathrm{RN}_{1}$ and $\mathrm{RN}_{4}$ ) samples produced amplicons of the expected size of ca. 850 bp, with SEOV R22 used as the positive control. cDNA from both amplicons was sequenced and showed the highest level of similarity (97\%) to Seoul hantavirus IR461 (GenBank accession no. AF329388) in a BLAST analysis. Further evidence the virus was Seoul-like was obtained through virus culture of homogenised lung from RN4. Infected Vero E6 cells (ECACC Vero C1008, clone E6) showed positive staining to a commercial monoclonal antibody (Progen, Germany) against the nucleocapsid protein of SEOV R22 strain after ten days in culture. After a further three passages the majority of cells demonstrated positive staining (Figure 1), the cells were harvested and virus isolated as previously described [2]. The isolate was designated Seoul hantavirus, strain Humber. Attempts to culture virus from RN1 were unsuccessful.

\section{Molecular analysis}

Hantaviruses are enveloped viruses with a tripartite, negative-strand RNA genome encoding the small (S), medium (M) and large (L) segments. Following multiple nucleotide sequence alignments of available SEOV sequences in GenBank, primers were designed for each segment spanning overlapping intervals of ca. $500 \mathrm{bp}$. Between two and seven independent runs were completed for each amplicon with $100 \%$ sequence conformity. Complete sequences for all three segments (RN1) were generated using standard Sanger sequencing on 


\section{FIGURE 1}

Vero E6 cells infected with Seoul hantavirus, strain Humber showing positive immunofluorescence, United Kingdom, October 2012

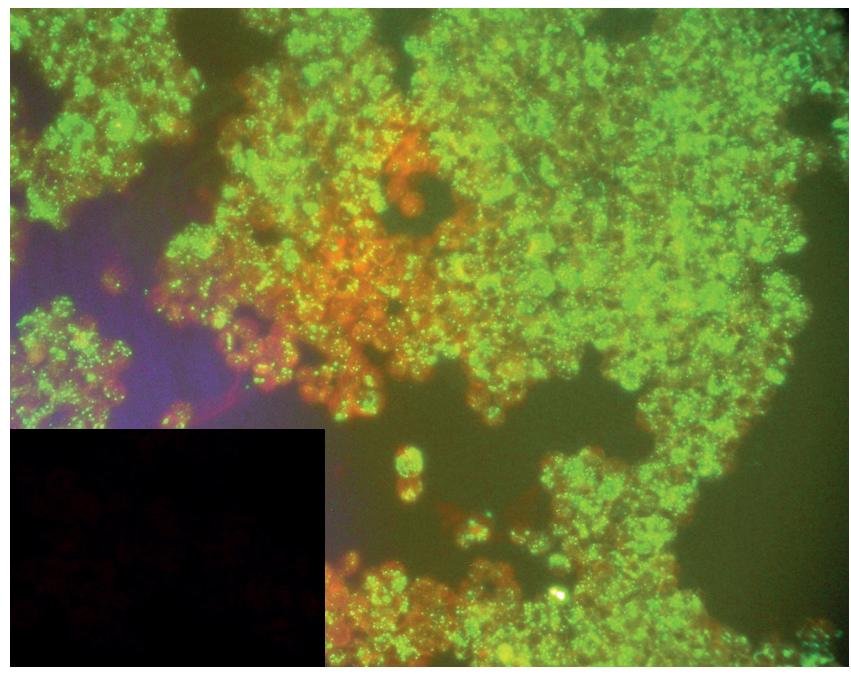

Negative and infected cells were stained with mouse monoclonal anti-Seoul N-protein antibody (Progen, Heidelberg, Germany) at 1:2 dilution, a secondary FITC-conjugated anti-mouse antibody at 1:64 (Sigma-Aldrich) and counterstained with $0.1 \%$ Evans blue. Viewed at 20x magnification under ultraviolet light; scattered, granular, punctate fluorescence (green) when compared to negative control Vero E6 cells only (inset, red) signified a positive reaction.

\section{FIGURE 2}

Phylogenetic analysis of the complete S segment of Seoul hantavirus, strain Humber, United Kingdom, October 2012

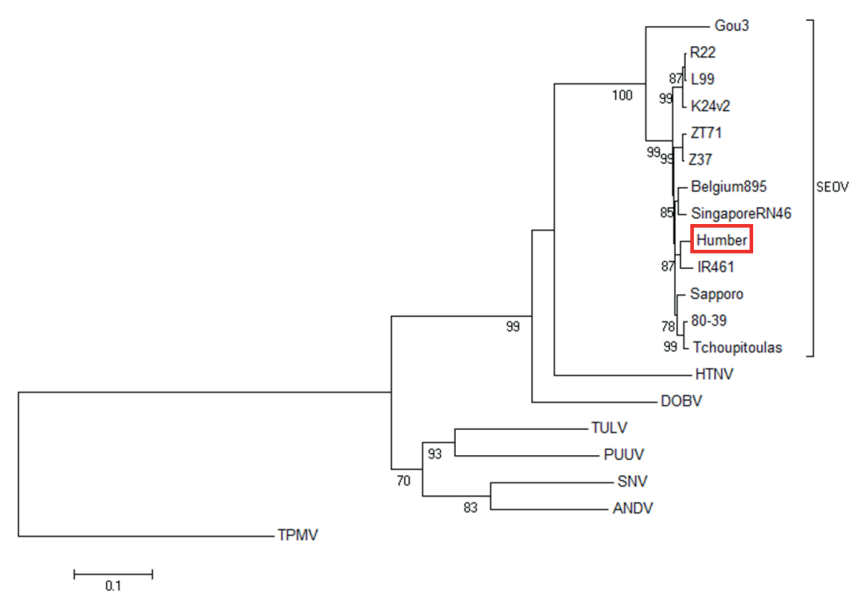

Horizontal distances represent the number of nucleotide differences. Bootstrap confidence limits exceeding $70 \%$ are shown for each branch node. Accession numbers for sequences extracted from GenBank: SEOV Gou3 AF288651, IR461 AF329388, K24V2 AF288655, R22 AF288295, L99 AF488708, Sapporo M34881, 80-39 NCo05236, SingaporeRN46 GQ27495, ZT71 AY75171, Z37 AF187082, DOBV JF920150, HTNV AB620031, TULV Z49915, PUUV AB433845, SNV L2578, ANDV AF291702 and AY 526097 . a $3130 x$ l sequencer (Life Technologies); they comprised $1,768 \mathrm{nt}, 3,651 \mathrm{nt}$ and 6,530 nt, each encoding one open reading frame. These sequences were deposited in the GenBank database under accession numbers JX879768-70 designated as Seoul virus, strain Humber. Sequence data from RN4 were compared with deposited sequence data from $\mathrm{RN}_{1}$, this demonstrated $1 \mathrm{nt}$ difference each in the $S$ and $M$ segments at positions 1,264 and 2,458 respectively.

The MEGA5 programme suite [3] was used to perform alignments using ClustalW and phylogenetic analysis. Phylogenetic trees were constructed from complete SEOV sequences using the neighbour-joining method, with bootstrap values obtained with 2,000 replicates. Subsequent genetic analysis of the $S$ and $M$ segments (RN1) confirmed their similarity to SEOV IR461 (Figures 2 and 3), with 63 (3.6\%) and 149 (4.1\%) nucleotide substitutions, respectively. The high homology and grouping of SEOV Humber with SEOV IR461 is surprising as IR461 is a distinctive strain [4] linked only to infections in laboratory workers in the UK and Belgium $[5,6]$. IR461 has not been detected in wild rodents.

Sequence data are not currently available for the $L$ segment of SEOV IR461, however enough L segment sequences are available from isolates in the SEOV group to show that the $L$ segment of the Humber strain (RN1) also clusters within the SEOV phylogenetic group of the hantavirus family (Figure 4).

\section{Discussion}

Hantaviruses (genus Hantavirus, family Bunyaviridae) are a group of rodent-borne viruses with a wide global distribution. Human infection most often occurs when breathing in dried aerosolised excreta from infected rodents. Two clinical syndromes are associated with severe disease: haemorrhagic fever with renal syndrome (HFRS) and hantavirus cardiopulmonary syndrome (HCPS) [7]. In the UK there has been growing evidence for human and animal exposure to hantaviruses demonstrated by the detection of specific antibodies and classic HFRS disease.

As hantaviruses are wholly associated with their rodent and insectivore hosts, their distribution is limited to that of their respective host species. SEOV is an exceptional hantavirus in that it has a global distribution owing to the dispersal of its carrier host, rats, through global trade. Outside of Asia, SEOV has been confirmed by molecular methods in rats in Africa [8], the Americas [9] and Europe [10-12]. There are several ports located on the Humber estuary including: the UK's largest port by metric tons, Grimsby and Immingham, Kingston upon Hull, and the UK's most inland port, Goole. It is possible that the importation of infected rats has led to the establishment of SEOV in local populations of $R$. norvegicus in the Yorkshire and Humber region. Few acute human cases caused by infection with SEOV have been confirmed outside of Asia [13] and none have simultaneously reported identification 


\section{FIGURE 3}

Phylogenetic analysis of the complete M segment of Seoul hantavirus, strain Humber, United Kingdom, October 2012

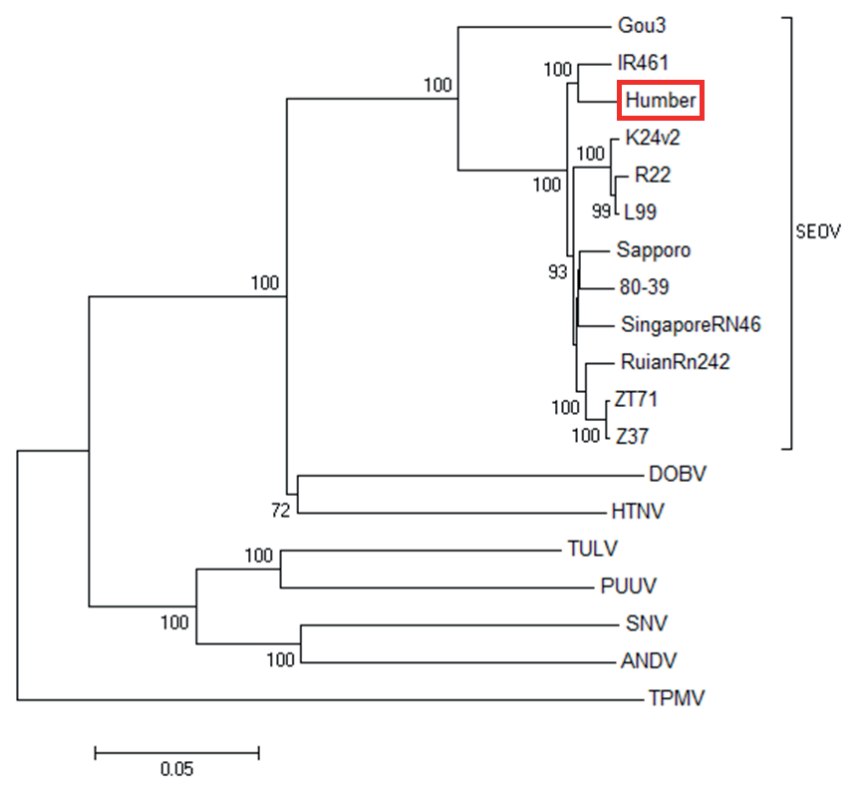

Horizontal distances represent the number of nucleotide differences. Bootstrap confidence limits exceeding $70 \%$ are shown for each branch node. Accession numbers for sequences extracted from GenBank: SEOV Gou3 AF145977, IR461 AF458104, K24V2 AF288654, R22 S68035, L99 AF288298, Sapporo M34882, 80-39 S47716, SingaporeRN46 GQ274943, RuianRn242 GU592928, ZT71 EF117248, Z37 AF190119, DOBV JF920149, HTNV AB620032, TULV NCo05228, PUUV AB433852, SNV NCo05215, ANDV AF291703 and TPMV NC010708. of the causative virus from rodents. Taking into consideration the patient's AKI, typical of SEOV, the high titre of antibodies detected specifically to the SEOV/HTNV group, the isolation of the virus from $R$. norvegicus from the patient's property, and its similarity to other pathogenic SEOV strains, it is highly likely the Humber strain is pathogenic to humans.

Due to the high levels of cross-reactivity between hantavirus species and the lack of viral detection in any published UK study, it has previously been impossible to confirm and identify the presence of a hantavirus in the UK. This represents the first isolation of a UK hantavirus from wild rodents and further confirmation of SEOV as a human pathogen outside of Asia. Given that $R$. norvegicus are ubiquitous in the UK, research is ongoing to determine the extent of human exposure to this virus in the region. Furthermore, with serological evidence specifically for SEOV previously reported in rats in Northern Ireland [14], it is unlikely this virus is restricted to north-east England.

\section{Conclusion}

Here we report the first detection and characterisation of a UK strain of Seoul hantavirus from wild rats. We feel it is important to raise awareness of hantavirus as a potential cause of renal disease in the UK, as Seoul hantaviruses are capable of causing moderate HFRS and we have strong indications this virus is linked to human disease. Our findings confirm the existence of a hantavirus in the UK and will allow further studies to evaluate its prevalence as a human pathogen.

\section{Acknowledgments}

We would like to acknowledge and thank the patient and family for allowing us access to their property; Autilia Newton, North Yorkshire \& Humber Health Protection Unit, for her role in the ongoing investigation; and Kate Adams and Rolf Meigh, Hull and East Yorkshire NHS Trust, for clinical samples.

This work is produced by L.J Jameson under the terms of Doctoral research training fellowship issued by the NIHR. The views expressed in this publication are those of the author(s) and not necessarily those of the NHS, The National Institute for Health Research or the Department of Health. 


\section{References}

1. Puthavathana P, Lee HW, Kang CY. Typing of Hantaviruses from five continents by polymerase chain reaction. Virus Res. 1992;26(1):1-14

2. Lee HW. Virus Isolation. In: Lee HW, Calisher $\mathrm{CH}$, Schmaljohn CS, editors. Manual of hemorrhagic fever with renal syndrome and hantavirus pulmonary syndrome. Seoul: World Health Organization Collaborating Center for Virus Reference and Research (Hantaviruses); 1998. p. 74-9.

3. Tamura K, Peterson D, Peterson N, Stecher G, Nei M, et al. MEGA5: Molecular Evolutionary Genetics Analysis using Maximum Likelihood, Evolutionary Distance, and Maximum Parsimony Methods. Mol Biol Evol. 2011;28(10):2731-9.

4. Shi X, McCaughey C, Elliott RM. Genetic Characterisation of a Hantavirus Isolated from a Laboratory-Acquired Infection. J Med Virol. 2003;71(1):105-9.

5. Lloyd G, Bowen ET, Jones N, Pendry A. HFRS outbreak associated with laboratory rats in UK. Lancet. 1984;323(8387):1175-6.

6. Desmyter J, LeDuc JW, Johnson KM, Brasseur F, Deckers C, Ypersele SC. Laboratory rat associated outbreak of haemorrhagic fever with renal syndrome due to Hantaan-like virus in Belgium. Lancet. 1983;322(8365):1445-8.

7. Krüger DH, Schönrich G, Klempa B. Human pathogenic hantaviruses and prevention of infection. Hum Vaccin. 2011;7(6):685-93.

8. Lee HW. Hantaviruses: An emerging disease. Phil J Microbiol Infect Dis. 1996;25(1):S19-S24.

9. Yanagihara R. Hantavirus Infection in the United States: Epizootiology and Epidemiology. Rev Infect Dis. 1990;12(3):449-57.

10. Heyman P, Plyusnina A, Berny P, Cochez C, Artois M, Zizi M, et al. Seoul hantavirus in Europe: first demonstration of the virus genome in wild Rattus norvegicus captured in France. Eur J Clin Microbiol Infect Dis. 2004;23(9):711-7.

11. Filipe AR, Andrade HR, Sommer AI, Traavik T. Hantaviral antigens and antibodies in wild rodents in Portugal. Acta Virol. 1991;35(3):287-91.

12. Plyusnina A, Heyman P, Baert K, Stuyck J, Cochez C, Plyusnin A. Genetic characterization of Seoul hantavirus originated from Norway rats (Rattus norvegicus) captured in Belgium. J Med Virol. 2012;84(8):1298-303.

13. Vaheri A, Henttonen H, Voutilainen L, Mustonen J, Sironen T, Vapalahti 0 . Hantavirus infections in Europe and their impact on public health. Rev Med Virol. 2012; doi:10.1002/rmv.1722.

14. McKenna P, Clement J, Matthys P, Coyle PV, McCaughey C. Serological evidence of Hantavirus disease in Northern Ireland. J Med Virol. 1994;43(1):33-8. 